\author{
Csaba Földes \\ ORCID: 0000-0002-4711-2072 \\ Universität Erfurt
}

DOI: $10.19195 / 0435-5865.144 .12$

\title{
Kontaktvarietäten des Deutschen im Ausland. Bausteine und Impulse für eine vergleichende Sprachinselforschung
}

\begin{abstract}
s
Der vorliegende Text setzt sich mit der aktuellen Situation der Forschungen zu deutschen Kontaktvarietäten im nicht-deutschsprachigen Ausland auseinander. Grundlage der Betrachtungen bildet die dritte „German Abroad“-Tagung, die unter dem Titel „Kontaktvarietäten des Deutschen im Ausland" im März 2018 an der Universität Erfurt stattfand und in enger Verbindung mit dem Projekt „Ungarndeutsches Zweisprachigkeits- und Sprachkontaktkorpus“ (UZSK) steht, das am Lehrstuhl für Germanistische Sprachwissenschaft der Universität Erfurt bearbeitet wird. Ein zentrales Ziel des Beitrags ist es, Impulse zur Perspektivenerweiterung sowie zur Methodengenerierung im behandelten Problembereich zu geben, auf dem Weg zur paradigmatischen Etablierung einer vergleichenden Sprachinselforschung im internationalen Maßstab.
\end{abstract}

Schlüsselwörter: Sprachinseln, Deutsch im Ausland, Sprachvariation, Sprachkontakt, Mehrsprachigkeit, Dialekte, Sprachverlust

\section{Contact varieties of German abroad: Building blocks and impulses for a comparative language island research}

The text at hand is dealing with the present situation regarding research of German contact varieties in non-German-speaking countries. The observations are based on the 3rd "German Abroad" conference, which took place in March 2018 at the University of Erfurt under the title "Contact Varieties of German Abroad". The conference was also closely connected to the project "Corpus of bilingual speech and language contact by speakers of German varieties in Hungary" (UZSK) associated with the Chair of German Linguistics of the University of Erfurt. A pivotal goal of this paper is to suggest new ideas and approaches for the broadening of perspectives as well as the development of new 
methods in the discussed problem areas towards to the paradigmatic establishment of a comparative language island research on an international scale.

Keywords: language islands, German abroad, language variation, language contact, multilingualism, dialects, language loss

CsabaFöldes, UniversitätErfurt,LehrstuhlfürGermanistischeSprachwissenschaft, NordhäuserStr.63, 99089 Erfurt, Deutschland, E-Mail: csaba.foeldes@uni-erfurt.de

Received: 19.09.2018, accepted: 8.04.2019

\section{Gegenstand}

Unter dem Titel „Kontaktvarietäten des Deutschen im Ausland“ fand vom 8. bis 10. März 2018 an der Universität Erfurt die dritte „German Abroad“-Tagung statt, ${ }^{1}$ die vom Lehrstuhl für Germanistische Sprachwissenschaft sowie der Forschungsstelle für Interkulturalität und Mehrsprachigkeit (FIM) - nicht zuletzt in Verbindung mit dem Forschungsprojekt Ungarndeutsches Zweisprachigkeits- und Sprachkontaktkorpus (UZSK) - veranstaltet wurde. ${ }^{2}$

\section{Thematischer Kontext}

Die Beschäftigung mit dem Realitätsbereich ,sprachlicher und kultureller Kontakt ${ }^{*}$ hat eine bemerkenswerte Tradition. Bereits Wilhelm Grimm formulierte: „Sobald aber Völker sich äußerlich nähern, so erfahren auch ihre Sprachen eine notwendige Wechselwirkung“ (Grimm 1986: 2017). ${ }^{3}$ Dieser Grundgedanke hat noch heute Berechtigung. Ebenfalls eine lange Historie haben Fragen nach Sprachwandel und Wechselwirkungen zwischen Sprachen in Kontakt, die schon im 19. Jahrhundert im Denkrahmen der historisch-vergleichenden Sprachwissenschaft als Forschungsthe-

${ }^{1}$ Die im Rahmen des Internationalen Forschungs- und Nachwuchsförderungsnetzwerkes (IFNIG) durchgeführte Tagung wurde freundlicherweise von der DFG gefördert. Den beiden Tagungssekretärinnen, Frau Dr. Bianka Burka-Rauhut und Frau Renáta Péter-Szabó, M.A., sei für die Mitwirkung beim Tagungsmanagement und Herrn Johannes Steudel, M.Ed., wissenschaftlicher Mitarbeiter am Lehrstuhl, für die Unterstützung bei der Erstellung dieses Berichts herzlich gedankt.

2 Der Gegenstandsbereich des Projekts ist eine Mehrsprachigkeitskultur mit spezifischen Ausprägungsstrukturen und Verwendungsmustern des Deutschen; es richtet sich zum einen auf die variations- und kontaktlinguistische Erforschung ungarndeutscher mündlicher Sprechhandlungen im interaktiven Alltag der Verständigung und zum anderen auf die Erstellung eines webbasierten Portals inklusive Datenbank für authentische ungarndeutsche Diskurse in Form von Tonaufnahmen, Transkripten und Texten. Projekthomepage: www.uzsk.de. Der Beauftragten der Bundesregierung für Kultur und Medien danken wir für die Förderung vielmals.

${ }^{3}$ Zur Historie und besonders zur wissenschaftlichen Verortung und Konzeptualisierung der Kontaktlinguistik vgl. Földes (2010). 
ma entdeckt wurden. Unzweifelhaft können Sprach(varietät)en infolge von Interaktionshandlungen zwischen Angehörigen verschiedener Kommunikationsgemeinschaften vielfältige Einflüsse aufeinander ausüben, was sich durch deren zahlreiche Erscheinungsformen auf verschiedenen sprachlichen Ebenen belegen lässt und als ein natürliches Produkt inter- bzw. transkultureller sozialer Prozesse aufzufassen ist. Mit der Erforschung derartiger Kontaktmanifestationen haben sich in den letzten Jahrzehnten im Bereich der Linguistik - sowohl aus diachronem als auch aus synchronem Blickwinkel - bereits mehrere Forschungsrichtungen z.T. unter Einsatz interdisziplinärer Methodeninventare auseinandergesetzt, was auf die Vielfalt und die Mehrdimensionalität möglicher Analysegesichtspunkte hinweist.

In diesem Zusammenhang ist auch die deutsche Sprache als Kontaktsprache vielfach in den Blickpunkt gerückt. Bedingt durch die Lage des deutschsprachigen Raums in Mitteleuropa und die lange zentraleuropäische Grenze ergaben und ergeben sich zahlreiche potenzielle Sprachkontaktsituationen zu verschiedenen Kontaktsprachen. Infolge von Auswanderungsbewegungen verschiedener Art entstand zudem eine Vielzahl von Kontaktkonstellationen mit dem Deutschen als Kolonial-, Migranten- oder Minderheitensprache sowohl innereuropäisch (z.B. in Rumänien oder Ungarn) als auch weltweit (z.B. Texasdeutsch in den USA, Hunsrückisch in Brasilien oder Namdeutsch in Namibia). Außerdem birgt auch der deutsche Sprach- und Kulturraum Potenzial für Sprachkontakt, wie etwa die autochthone Minderheitensprache Sorbisch in Brandenburg und Sachsen oder das Vorhandensein von allochthonen Migrantensprachen wie Türkisch, Spanisch oder Arabisch innerhalb Deutschlands. In summa kann Deutsch damit als wohl kontaktfreudigste Sprache Europas charakterisiert werden.

\section{Wissenschaftliche Vorgeschichte}

Diese Kontaktsituationen sind in der Linguistik - auch in jüngerer Vergangenheit - immer wieder aufgegriffen worden. Um den Diskurs zwischen den Akteuren anzuregen, Erkenntnisse auszutauschen und Schritte in Richtung einer vergleichenden Sprachkontaktforschung zu unternehmen, wurde 2014 die Tagungsreihe „German Abroad“ ins Leben gerufen, die auf die deutsche Sprache und ihre Variationsbreite in Mehrsprachigkeitskonfigurationen außerhalb des deutschen Sprachraums fokussiert.

Die Tagungen der Reihe finden alle zwei Jahre statt, den Auftakt machte im Juli 2014 die erste German-Abroad-Tagung am Institut für Germanistik der Universität Wien. Unter dem programmatischen Titel „Perspektiven der Variationslinguistik, Sprachkontakt- und Mehrsprachigkeitsforschung“ wurden in erster Linie Varietäten des Deutschen in Nord- und Südamerika behandelt, zudem wurden Einzelbeispiele aus Norditalien, Polen, Tschechien und dem Pazifikraum 
vorgestellt. ${ }^{4}$ Zwei Jahre später war die University of Texas in Austin Gastgeber der zweiten Konferenz, Hauptschwerpunkt war ebenfalls der amerikanische Kontinent, ergänzt mit einzelnen Beiträgen zu Namibia, Ozeanien, Russland und Ostmitteleuropa.

\section{Konzept und Ziele}

Der Komplexität und der Spezifität der linguistischen Annäherungen sollte auf der dritten German-Abroad-Tagung eine konstitutive Rolle zukommen. Ein zentrales Ziel war es, charakteristische Merkmale von Kontaktvarietäten der deutschen Sprache aufzuzeigen und zu beschreiben, wobei der Aspekt der Inter- bzw. der Transkulturalität eine vorrangige Rolle spielte. Somit war z.B. das Kommunikationsverhalten deutschsprachiger Gruppen bzw. Minderheiten außerhalb des zusammenhängenden deutschen Sprachgebiets in traditionellen „Sprachinseln“ und in sonstigen inter- bzw. transkulturellen Konfigurationen (etwa in Migrationskontexten) von hoher Relevanz - mit besonderem Blick auf Sprachstrukturen, Sprachverwendungsaspekte und sozialpsychologische Faktoren. Ebenso begrüßt wurde ${ }^{5}$ die Beschreibung deutscher Lernervarietäten bzw. ihre Anwendungsmöglichkeiten im Deutsch-/DaF-/DaZ-Unterricht sowie die Betrachtung anderer Sprachen und deren Varietäten, die unter dem Kontakteinfluss des Deutschen stehen und in verschiedenen Interaktionssituationen, Medien usw. erscheinen.

Im Erkenntnisinteresse der Konferenz lagen folglich Kontaktprozesse und -ergebnisse in diversen mündlichen oder schriftlichen Sprachverhaltensdomänen auf der morphosyntaktischen, lexikalisch-semantischen oder pragmatischen Ebene, d.h.:

- Sprachkontaktsituationen und -phänomene sowie bilinguale kommunikative Praktiken in ihrer Dynamik mit Blick auf deutschsprachige Gruppen außerhalb des deutschen Sprachraums;

- Kontaktinduzierte Variation bzw. Kontaktvarietät als Thema oder als Faktor im Deutsch-/DaF-/DaZ-Unterricht bzw. im deutschsprachigen Unterricht im Ausland;

- Spracheinstellungen und Sprachperzeptionen im Hinblick auf Kontaktvarietäten des Deutschen.

Die Tagungsreihe sollte mit dieser Konferenz hinsichtlich des Betrachtungsraums ausgebaut werden: Zum einen sollte der analytische Blick auf das gesamte Verbreitungsareal des Deutschen und seiner spezifischen Ausprägungen erweitert werden, sodass u.a. auch die traditionellen „Hochburgen“ des deutschen Sprachkontaktgeschehens im östlichen (Mittel-)Europa vertieft ins Blickfeld geraten

${ }^{4}$ Siehe ausführlicher den Tagungsband von Lenz (2016).

${ }^{5}$ Eine andere Frage ist, dass dieses Themenfeld in der Tagungsrealität deutlich weniger Raum einnahm. 
konnten. Zum anderen war es ein prominentes Anliegen, einer weitgehend multiund interdisziplinären Auseinandersetzung mehr Reflexionsraum zu bieten, um das Kontaktverhalten der deutschen Sprache in diversen Interaktionskonfigurationen unter möglichst vielfältigen Gesichtspunkten und dadurch schließlich integrativ zu erschließen.

\section{Tagungsablauf und Denkrichtungen}

Nach der Tagungseröffnung startete Claudia M. Riehl mit ihrem Plenarvortrag zu Sprachkontakt und -erosion den akademischen Austausch. Dabei zeigte sie anhand von generationenübergreifenden Studien zu Sprachinselvarietäten in Südaustralien, Südbrasilien und Russland Entwicklungen im Bereich von Sprachvereinfachungsprozessen am Beispiel des Kasusabbaus auf und konstatierte, dass der Kasusabbau in der Nominalphrase in allen Gruppen ähnlichen Entwicklungspfaden folgt, während im Pronominalsystem unterschiedliche Verläufe auszumachen sind. Simplifizierungs- und Restrukturierungsprozesse werden durch den schwindenden Gebrauch vorangetrieben. Zudem sind individuelle oder kontaktinduzierte Wandelphänomene, etwa der Wegfall des Artikels in Kontaktstellung mit Russisch, zu beobachten. Aus diesen Befunden leitete die Referentin Perspektiven für die künftige Forschung ab.

Nachfolgend wurden grundsatztheoretische und methodologische Kernfragen diskutiert. Hans Boas plädierte für einen neuen Ansatz zum systematischen Vergleich deutscher Sprachinseln. Dabei ging er grundsätzlich davon aus, dass zur Gegenüberstellung von Kontaktvarietäten Standarddeutsch als tertium comparationis genutzt wird, wenngleich eine Vielzahl von internen und externen Einflussfaktoren die Entwicklung der Varietäten beeinflusst. Da kein einheitlicher Rahmen für einen varietätenübergreifenden Vergleich besteht, ist dieser häufig kaum zu leisten. Boas schlägt daher vor, Konstruktionsgrammatik und Frame-Semantik als gemeinsamen theoretischen Rahmen zu verwenden und eine computergestützte Infrastruktur zum systematischen Sammeln und Vergleichen von Sprachinseldaten zu entwickeln. Im nächsten Referat ordnete Ulrich Ammon die aktuelle Verbreitung und Stellung der deutschen Sprache in der Welt ein und leitete daraus sprachenpolitische Konsequenzen ab. Als internationale und plurizentrische Sprache verfügt Deutsch nach Hochrechnung des Referenten weltweit über etwa 360 Millionen „Sprecher“ (?) - mehrheitlich ehemalige DaF-Lerner, die Deutsch oft kaum besser als auf A1-Niveau des GER beherrschen. Nichtsdestoweniger sieht Ammon die Position der deutschen Sprache im In- wie im Ausland gefährdet, insbesondere durch die Weltsprache Englisch. Mit Verweis auf die lange Tradition einer deutsch-stabilisierenden Sprachenpolitik im Ausland - etwa durch den DAAD, das Goethe-Institut, das deutsche Auslandsschulwesen oder das Institut 
für Auslandsbeziehungen - schlägt Ammon die Schaffung weiterer Anreize für das Deutschlernen vor, auch weil instrumentelle Motivation seiner Ansicht nach effektiver sei als die identifikatorische.

Die Varietät des Deutschen in Namibia, das sog. Namdeutsch, bildete den Reflexionsgegenstand mehrerer Vorträge. Zunächst gab Marianne Zappen-Thomson eine kurze Übersicht zur Geschichte des Deutschen in Namibia und bot einen Einblick in die Verwendung des Namdeutschen einschließlich ausgewählter Kontaktphänomene - v.a. Bedeutungsverschiebungen, In- und Transferenzen, aber auch Erscheinungsformen von Sprachökonomie und Analogie. Weitere Details gingen aus dem Referat von Horst Simon, Britta Stuhl und Christian Zimmer hervor; nämlich, dass durch Sprachkontakt neben lexikalischem Transfer auch grammatische Innovationen begünstigt werden. Mit dem Beispiel der über das Standarddeutsche hinausgehenden Entwicklung des $s$-Genitivsuffixes zu einem Klitikon (deine Ma-s Kleider) zeigten sie einige neuere Entwicklungen im Namibiadeutschen unter Rückgriff auf Sprachdaten aus ihrem elektronischen Korpus. Die aktuellen soziolinguistischen Verwendungsmodalitäten der Deutsch-Varietät in Namibia stand ebenfalls zur Diskussion: Heike Wiese und Yannic Bracke stellten ihre Forschung zur Registerdifferenzierung im Namdeutschen vor und wiesen darauf hin, dass sich besonders in informellen Gesprächen Abweichungen vom Standarddeutschen - häufigerer lexikalischer Transfer, aber auch Differenzen auf struktureller Ebene - ergeben, während das formelle Register dem Standarddeutschen ähnlich ist, allerdings auch Belege für eine eigene namibische Varietät zeigt. Henning Radkes Referat beleuchtete schließlich die Rolle computervermittelter Kommunikation zur Analyse kontaktsprachlich bedingter Phänomene im Namdeutschen. Im Rahmen der interaktionalen Soziolinguistik untersuchte Radke die durch vernetzte Mehrsprachigkeit entstandenen Schreibmuster der deutsch-namibischen Diaspora, zu der u.a. inter- und intrasententielle Kode-Umschaltung, typische Entlehnungen im namibischen Deutsch und Lehnübersetzungen aus dem Englischen und Afrikaans gehören.

Die Kontaktvarietäten des Deutschen in Ungarn wurden erstmals im Rahmen dieser Tagungsreihe ausführlich und multiperspektivisch betrachtet. Die Referierenden bedienten sich dabei verschiedener analytischer Blickwinkel und methodischer Zugänge: Nachdem sich die gesprochene Sprache der Minderheitensprecher in Ungarn als Forschungsobjekt relativ großer Beliebtheit erfreut, wandte sich Elisabeth Knipf-Komlósi nun einer geschriebensprachlichen Varietät und damit der Schriftkompetenz ungarndeutscher Dialektschreiber zu. Die empirische Basis dazu bildeten Ausschnitte aus dialektalen schriftlichen Textprodukten privater Art aus den 1970er, 1990er und 2000er Jahren. Die z.T. noch prospektiven Überlegungen richteten sich auf die Ermittlung einiger charakteristischer system- und soziolinguistischer Merkmale dieser Dialektdokumente. Koloman Brenner stellte phonologische Besonderheiten einer ungarndeutschen Dialektform in Westungarn vor. Mittels akustischer Analyse untersuchte er verschiedene Diphthongtypen hin- 
sichtlich lautlicher Parameter und verglich diese mit Daten der ungarischen Standardvarietät, um mögliche Inferenzphänomene sichtbar zu machen. Márta Müller und Réka Miskei präsentierten eine empirische Studie über die Einstellung Ungarndeutscher zu Deutschland bzw. zur deutschen Sprache.

Die große Bandbreite an Forschungsfragen und Zugriffen wurde auch an folgenden Vorträgen deutlich: Daniela Pelka befasste sich eingehend mit der Lexik im oberschlesischen Deutsch, indem sie in Personenbezeichnungen Kennzeichen für Alter, Körpergröße und -fülle analysierte. Die Präsentation von Stefaniya Ptashnyk demonstrierte, dass Sprachkontaktforschung auch mit einer historischen Ausrichtung produktiv sein kann: Im 19. Jahrhundert wurde das Lateinische an der Universität Lemberg zunehmend von Deutsch, Polnisch, Ukrainisch und Ruthenisch abgelöst. Der Beitrag hinterfragte, wie der Sprachenwechsel unter verschiedenen außersprachlichen Einflüssen vonstattenging. Edgar Baumgärtner befasste sich mit der deutschen Verbalmorphologie in der Altai-Region in Russland. Er wies nach, dass sich in den dort nur mündlich gebrauchten und keinen sprachnormierenden Instanzen unterliegenden Kontaktvarietäten Sprachwandelprozesse gut beobachten lassen. Bezogen auf die Verbalmorphologie deuten seine ersten Befunde in Einklang mit der Natürlichkeitstheorie auf Prozesse zur Regularisierung starker Verben. Mit germanischen und slawischen Einflüssen in obersorbischen Grammatiken des 19. Jahrhunderts beschäftigte sich hingegen Kai Witzlack-Makarevich, der das Auseinanderbrechen der Varietät in eine high und eine low variety diskutierte.

Den zweiten Tag eröffnete Hermann Scheuringer mit seinem Plenarvortrag zum (teilweise historischen) Mehrsprachen- und Kulturkontaktraum ,östliches sowie südöstliches Europa' und bilanzierte, dass das Deutsche - trotz z.B. der Weltkriegsfolgen - nach wie vor eine bedeutende Rolle spielt und den Multilinguismus in diesem Areal wie kaum eine andere Sprache prägt.

Den Fokus auf ostmitteleuropäische Deutsch-Varietäten verstärkend wurden auch Sprachkontaktkonstellationen in Rumänien intensiv diskutiert. Beispielsweise charakterisierte Sorin Gadeanu das gegenwärtige Deutsch in Westrumänien als „nachminderheitliche“ Sprache, d.h. eine Varietät an der Schwelle von L1 zu L2 und untersuchte dafür zwei Schulklassen - die eine mit überwiegend deutscher, die andere mit überwiegend rumänischer Unterrichtssprache und Deutsch-Intensivkurs. Einem ähnlichen Untersuchungsfeld wandte sich Delia Cotârlea zu. Sie erläuterte die Situation der Schriftsprache Deutsch im Kontext des Deutschunterrichts in rumänischen Schulen und kam zu dem Schluss, dass aufgrund der geringen kommunikativen Relevanz des Deutschen im außerschulischen Kontext die deutsche Sprache nicht mehr nach der L1-Didaktik unterrichtet werden sollte. Stattdessen plädierte sie für eine Änderung der Curricula hin zu einer DaF-Didaktik. Spezifische sprachliche Muster und Auffälligkeiten in der „Allgemeinen Deutschen Zeitung für Rumänien“ (ADZ) standen im Referat von Ioana H. Fierbințeanu im Blickpunkt, indem sich die Referentin der Frage 
widmete, inwieweit Formen konzeptioneller Mündlichkeit auftreten und welche Wirkungen sie erzielen.

Sigita Barniškienė unterzog die Lexik eines preußisch-litauischen Dialekts am Material eines Romantextes einer genaueren Prüfung und gliederte die Germanismen und Slawismen in semantische Felder, um den Benennungsbedarf zu systematisieren, die zur Eingliederung ins litauische Sprachsystem führten, beispielsweise im Bildungswesen, bei Speisen und Kleidung oder bezüglich Politik und Gesetzgebung. Aufgrund der Normierung der litauischen Sprache und nicht zuletzt der Folgen des Zweiten Weltkriegs wurden die einst integrierten Wörter allerdings mehrheitlich bereits verdrängt. Unter namenkundlichen Gesichtspunkten untersuchte Aneta Stojić den deutsch-kroatischen Sprachkontakt. Ihre Analyse machte deutlich, dass deutsches Wortgut sowohl in der standard- und substandardsprachlichen Lexik des Kroatischen als auch in dessen onomastischem Inventar eine bedeutende Rolle spielt: Aus deutschen Lehnwörtern entstanden Eigennamen, die heute zu den ältesten Anthroponymen und Toponymen in Kroatien gehören; deutsche Familiennamen haben sich als Eigennamen erhalten und sind in unterschiedlichem Grad assimiliert oder sind als latente Formen verborgen. Boris Blahak erforschte Sprachkontaktsituationen in Umfeld von Franz Kafka. Der Schriftsteller hatte ab 1911 versucht, Jiddisch aufgrund seiner jüdischen Herkunft in einem rezeptiven, an den L1-Erwerb angelehnten Prozess zu erlernen; Kafkas Fortschritt wird durch sein Tagebuch - verfasst in seiner Lernervarietät des Jiddischen - dokumentiert. Letztlich scheiterte sein Spracherwerb ebenso wie seine ,jüdische Spracherwerbstheorie“.

Einen weiteren thematischen Block verkörperten Grammatikalisierungen und Spracheinstellungen zu Varietäten des Deutschen. Péter Maitz und Lena-Marie Schmidtkunz stellten Ergebnisse ihrer Forschung zur deutschbasierten Kreolsprache ,Unserdeutsch“ vor, die belegen, dass Unserdeutsch einerseits - im Vergleich zum Standarddeutschen - über ein relativ elaboriertes Aspektsystem verfügt, dieses andererseits - für die Kontexte von Kreolsprachen - typische Strukturmerkmale aufweist. Den Verbaspekt betrachtete auch Adam Tomas in seiner Untersuchung zum Pennsylvaniadeutschen; er arbeitete heraus, dass die vom Englischen eingeschlossene Varietät ein - im Standarddeutschen deutlich höheren Restriktionen unterliegendes - produktives am-Progressiv-Paradigma entwickelt hat. Mit der Perspektive der Sprachinselforschung zur Begründung dieser Formen geht einher, dass diese in der Standardsprache offensichtlich nicht aus morphosyntaktischen, sondern aus soziolinguistischen Gründen keine Anwendung finden.

Den Betrachtungen von Matthias Fingerhuth über das Deutsche in Texas lagen zwei Positionen im Forschungsdiskurs zugrunde: Zum einen wird in der Sprachinselforschung traditionell eher gesprochene Sprache behandelt, zum anderen wird über das Deutsche als plurizentrische Sprache ins Feld geführt, dass einige Sprachinseln zumindest zeitweise ein funktionierendes geschriebensprachliches Druckwesen etablieren konnten. An ausgewählten Beispielen aus händischer 
Recherche in Korpora gedruckter texasdeutscher Zeitungen um 1900 belegt Fingerhuth, dass im Bereich der Lexik kontaktsprachliche Einflüsse existieren, die für das Vorhandensein von Varianten des Standarddeutschen in Texas - vielleicht sogar einer texanischen Standardvarietät des Deutschen - sprechen. Lucas Löff Machado beschäftigte sich mit der Perzeption des Deutschen in Brasilien. Durch Datenerhebung mittels Interview und anschließender Auswertung schlussfolgerte er, dass das Konzept „Hochdeutsch“ im Lauf der Immigration geprägt wird, wobei für die Aktualisierung der Sprachnorm Sprachkontakte mit weiteren Sprechergruppen, geliebten oder öffentlichen Bezugspersonen sowie unter kirchlicher Beteiligung wichtige Einflussfaktoren darstellen.

Mit dem Ziel einer flächendeckenden und systematisch vergleichbaren Dokumentation sprachlicher Strukturen und ihrer außersprachlichen Bedingungen tritt in Südbrasilien das Sprachatlas-Projekt ALMA auf. In Subprojekten sollen auch die einzelnen Dialekte der Zuwanderer abgebildet werden; so konnte bereits von den methodologischen Grundlagen für das Hunsrückische (ALMA-H) berichtet werden. Sebastian Kürschner widmete sich nun Vorüberlegungen zur Erfassung bairischer Varietäten in Südbrasilien (ALMA-B), etwa der Auswahl von Erhebungsorten, der Modifikation der Befragungsmethoden in Anpassung an bestehende dialektologische Projekte mit dem Kontext Bairisch sowie der Auswahl eines Forschungsteams. Mit Deutsch in China beschäftigte sich Michael Szurawitzki, der die Werbesprache im Shanghaier Exilperiodikum „Gelbe Post“ in den 1930er Jahren auswertete. Im Fokus seiner Analyse standen quantitative und topikalische Aspekte sowie Textsorten-Charakteristika: Häufig referiert die Werbung auf die Heimat der Exilgemeinde und orientiert sich zumindest teilweise an deren (schwierigem) Alltag. Die sprachkommunikative Heterogenität innerhalb von Migrantengemeinschaften demonstrierten Eva Duran Eppler, Christoph Gabriel und Jonas Grünke: Ausgehend von den Beobachtungen, dass Mehrsprachige bei der Kode-Umschaltung oft zwischen Artikelwort und Nomen die Sprache wechseln und dass sprachliche Kategorien in Kontaktsituationen konvergieren können, werteten sie ein historisches lautsprachliches Korpus von in London lebenden jüdischen Emigranten aus Wien in Bezug auf die Realisierung der Schwa-Artikel aus. Ihre Erwartung phonetisch konvergierter Schwa-Realisierungen bestätigte sich zwar nur teilweise, doch zeigten die Befunde unterschiedliche Strategien der Fremdsprachenaneignung (L1-Transfer bzw. target overshoot) sowie Anzeichen von Spracherosion bei Teilen der Sprecher. Dies legt den Einbezug außersprachlicher Informationen bei der Interpretation phonetischer Messdaten nahe.

Schließlich wurden auch Fragen nach der sprachlichen Identität innerhalb deutschsprachiger Gemeinschaften im Ausland erläutert. Patrick Wolf-Farré rückte die bisher kaum untersuchten Deutschchilenen in den Mittelpunkt. In Chile wirken gegenläufige Kräfte: Einerseits ist der Gebrauch des Deutschen als Umgangssprache insgesamt stark rückläufig, andererseits verfügt es über eine starke institutionelle Sicherung und ein hohes Prestige; dementsprechend wirken Faktoren wie 
schulische Bildung oder die Neuen Medien einem vollkommenen Verschwinden der deutschen Sprache entgegen. Aufgrund dessen bilanzierte der Referent, dass die Deutschchilenen keine Sprachinsel darstellen, wohl aber einen aus der Sprachinsel hervorgegangenen Teil der chilenischen Gesellschaft. Aufgrund der Verschiedenheit der Faktoren bzw. deren Relevanz für einzelne Sprecher plädierte er für einen gemischten Einsatz sowohl quantitativer als auch qualitativer Erhebungsverfahren, um individuelle Sprachbiographien in die Beschreibung der gesamten Sprechergemeinschaft mit einfließen zu lassen. Sheena Shah und Erika Herrmann stellten eine deutschsprachige Gemeinschaft im ländlichen Südafrika vor. Diese Varietät wird in Kroondal von der Mehrheit der Dorfbewohner als Erstsprache gesprochen und ist die Unterrichtssprache in der Schule. Mittels qualitativer Fragebögen, Interviews und Gruppendiskussion arbeiteten Shah und Herrmann heraus, dass man innerhalb der Dorfgemeinschaft stolz darauf ist, die deutsche Sprache zu sprechen und dass die Sprache ein starkes Zusammengehörigkeitsgefühl konstituiert.

Der letzte Konferenztag begann mit dem Plenarvortrag von Cléo V. Altenhofen, der sich ausführlich mit den ungefähr 14 Einwanderervarietäten des Deutschen in Brasilien auseinandersetzte und die Hypothese ableitete, dass das ursprünglich aus dem westmittelhochdeutschen Sprachraum stammende Hunsrückische unter den Deutschvarietäten in Brasilien die Rolle einer vermittelnden Sprache zwischen sehr verschiedenen Dialekten einnimmt, die aber dennoch über großen Variantenreichtum verfügen.

Im Fokus standen nachfolgend Varietäten des Deutschen in Russland. Nina Berend und Elena Frick gaben einen Überblick über die Verbreitung des Russlanddeutschen weltweit und dessen verschiedenartige Entwicklung im Kontakt mit anderen Sprachen. Gleichzeitig konstatierten sie einen ,plötzlichen Stillstand“ in Russland, ausgelöst durch Massenauswanderungen innerhalb weniger Jahre. Ihre Ausführungen liefen auf die Darstellung ihrer Korpus-Datenbank Ru-Di-Dat am Institut für Deutsche Sprache (IDS) in Mannheim hinaus und thematisierte Herausforderungen, die durch unterschiedliche Ausprägungsformen des Russlanddeutschen entstehen könnten, wenn man die im Internet freigegebenen Sprachinseldaten für vergleichende Analysen heranzieht. Morphosyntaktische Variation in gesprochenen Kontaktvarietäten in Sibirien wurden von Christiane Andersen in den Blick genommen. Ihre Untersuchung von Nominalphrasen mit einer substantivischen Verwandtschaftsbezeichnung weist als Zwischenergebnis einerseits auf große Variantenvielfalt, andererseits auf die Reduktion lexikalischer Mittel und syntaktischer Funktionen hin. Ebenfalls in Krasnojarsk (Sibirien) ist das Forschungsunterfangen von Shanna Sershanowa zum Redeverhalten von Frauen und Männern in deutschen Dialekten angesiedelt, das deutliche Geschlechterunterschiede aufweist: Frauen beherrschen den Dialekt besser und nutzen ihn häufiger, auch inhaltlich lassen sich Unterschiede - etwa bei der Wahl der Gesprächsthemen - feststellen. 
Jan Wirrer dokumentierte den Restbestand des Niederdeutschen im Mittleren Westen der USA. Unter Nutzung von sprecherbiographischen Interviews, freien Erzählungen und Übersetzungstests erhob er Sprachdaten, mit denen er eine Vielzahl von Sprachkontaktphänomenen auf allen sprachlichen Ebenen ermittelte. Hannes Scheutz stellte schließlich einen „sprechenden“ Atlas zu Austriazismen und Germanismen in kroatischen Dialekten vor, der ein breites Spektrum an Lehnwörtern aus dem Deutschen enthält. Die Entlehnungen decken ein weites zeitliches Spektrum ab; der Referent wies sowohl Bezüge zu den Habsburgern als auch zur heutigen Zeit nach.

Offiziell beendet wurde die Tagung mit einer resümierenden Abschlussdiskussion, in der - neben der inhaltlichen Zusammenfassung der Ergebnisse - u.a. die Weiterführung der Tagungsreihe beschlossen und die kommenden Aufgaben besprochen wurden. Als Veranstalter der Tagung benannte Csaba Földes abschließend mit Marianne Zappen-Thompson eine würdige Nachfolgerin, die damit Gastgeberin der vierten German-Abroad-Tagung im März 2020 in Windhoek (Namibia) wird. ${ }^{6}$

\section{Beitrag der Konferenz zur Tagungsreihe}

Neben den informativen Beiträgen und anregenden Diskussionsbeiträgen kann die Tagung im „Erfurter Aufruf zur Sicherung von Sprachinseldaten“ ein weiteres bedeutsames Ergebnis vorweisen: Im Vorfeld der Tagung wurde der Aufruf von Hans C. Boas, Csaba Földes, Claudia M. Riehl, Thomas Schmidt und Heike Wiese als Diskussionsgrundlage vorbereitet und auf der Tagung in Erfurt erstmals vorgestellt und erörtert. Anliegen des Aufrufs ist es, die große Vielfalt der in den letzten 50 bis 70 Jahren erhobenen Sprachdaten dauerhaft und systematisch zu archivieren, um einem Verlust dieser Daten vorzubeugen. $\mathrm{Zu}$ diesem Zweck wird vorgeschlagen, in Anlehnung an schon bestehende Projekte innerhalb der German-Abroad-Community ${ }^{7}$ eine einheitliche Infrastruktur zur Digitalisierung, Aufbereitung und Archivierung von Daten aufzubauen. In einem ersten

${ }^{6}$ Das wissenschaftliche Programm rundeten eine germanistische Stadtführung in Erfurt sowie am Folgetag eine Exkursion auf einen Teilcampus der Universität, das Forschungszentrum Gotha der Universität Erfurt in Schloss Friedenstein, ab. Basierend auf der Tagung ist eine Buchpublikation vorgesehen: Die eingereichten Manuskripte werden doppelblind begutachtet und die positiv evaluierten sollen demnächst in einem hochwertigen Sammelband mit dem Titel „Kontaktvarietäten des Deutschen im Ausland“ in der Schriftenreihe Beiträge zur Interkulturellen Germanistik (BIG) beim Verlag Narr Francke Attempto in Tübingen erscheinen.

${ }^{7}$ Beispielsweise kooperiert das Archiv für Gesprochenes Deutsch am IDS mit dem Texas German Dialect Project an der University of Texas at Austin, um eine angemessene Infrastruktur zur Digitalisierung, Aufbereitung und Archivierung von bestehenden Sprachinseldaten aufzubauen (Näheres: https://tgdp.org/). Außerdem wird z.B. im Rahmen des unter Abschnitt 1 erwähnten Forschungsprojekts Ungarndeutsches Zweisprachigkeits- und Sprachkontaktkorpus (UZSK) an der 
Schritt sollen die vorhandenen Bestände gesichtet werden, für die Inventarisierung steht eine dafür eingerichtete Kontaktstelle zur Verfügung. Auf der nächsten German-Abroad-Tagung 2020 soll dann die weitere Vorgehensweise vereinbart werden, beispielweise wie die Daten einheitlich aufbereitet werden können, um sie nicht nur zu sichern, sondern auch um für eine (digitale) vergleichende Sprachinselforschung nutzbar zu machen und damit die Forschungsdisziplin selbst zu innovieren oder wie solche Mengen an Daten möglichst aufwandsminimal und nachhaltig gespeichert werden können. Mit diesem Ziel sind die Teilnehmer(innen) der Tagung auch aufgefordert, anderen Akteuren die Bedeutung des Anliegens $\mathrm{zu}$ verdeutlichen und Kooperationspartner zu gewinnen. Das Vorhaben stieß auf breite Zustimmung - der Aufruf wurde zudem von den meisten Teilnehmenden unterzeichnet - und wird nun konkretisiert.

Resümierend gilt, dass mit der Konferenz in Erfurt die Tagungsreihe eine quantitative und qualitative Erweiterung erfuhr: Während sich die Größenordnung der Vorgänger-Veranstaltungen auf etwa 20 Referate erstreckte, konnten nunmehr von den zahlreichen Vortragsangeboten interessierter Kolleg(inn)en 39 qualitativ hochwertige Abstracts angenommen werden. An der Tagung nahm die Mehrheit der renommiertesten Wissenschaftler(innen) der germanistischen Sprachinselforschung aus 17 Ländern von vier Kontinenten persönlich teil, was einen hohen wissenschaftlichen Standard garantierte. Ebenso international waren die arealen Betrachtungsobjekte der Tagung: Einerseits wurden, wie bei den vorherigen Konferenzen, Sprachkontaktsituationen mit Deutsch auf dem amerikanischen Kontinent eingehend thematisiert, andererseits galt mit jeweils mehreren Fachvorträgen auch dem ostmittel- bzw. südosteuropäischen Sprachraum (Ungarn, Rumänien) sowie Russland und Namibia ein besonderes Augenmerk. Zudem wurde der inhaltliche Horizont der Konferenz nicht lediglich bezogen auf vielfältigere sprachliche und soziokulturelle Konfigurationen erweitert, sondern auch auf differente Wissenschaftskulturen und deren Vorgehensweise bei der Erfassung von Mehrsprachigkeits- und Sprachkontaktsituationen. Dies bedeutet, dass die Referierenden nicht nur aus unterschiedlichen linguistischen Teildisziplinen und Paradigmen kamen, sondern auch verschiedene national geprägte Diskurs- und Argumentationstraditionen (amerikanische, slawische etc.) vertraten. Schließlich haben es die differenten Gegenstände und Zugänge ermöglicht, sich mit den einschlägigen Facetten der variationsfreudigen deutschen Sprache breitgefächert und interdisziplinär auseinanderzusetzen. Somit konnten produktive Impulse zur Perspektivenerweiterung sowie zur Methodengenerierung im behandelten Problembereich gegeben werden - auf dem Weg zur paradigmatischen Etablierung einer vergleichenden Sprachinselforschung im internationalen Maßstab, zu der auch unser UZSK-Projekt (siehe Abschnitt 1) einen Beitrag leisten soll.

Universität Erfurt authentisches Sprachinsel-Material erfasst, digital archiviert und für die Öffentlichkeit zugänglich gemacht (Näheres: www.donauschwaebisch.de). 


\section{Literatur}

Földes, Csaba (2010): Was ist Kontaktlinguistik? Notizen zu Standort, Inhalten und Methoden einer Wissenschaftskultur im Aufbruch. In: Bergmann, Hubert / Glauninger, Manfred Michael / Wandl-Vogt, Eveline / Winterstein, Stefan (Hrsg.): Fokus Dialekt. Analysieren - Dokumentieren - Kommunizieren. Festschrift für Ingeborg Geyer zum 60. Geburtstag. Hildesheim/Zürich/ New York. (= Germanistische Linguistik; 199-201/2010). S. 133-156.

Grimm, Wilhelm (1986): Bericht über das Deutsche Wörterbuch (1846). In: Reiher, Ruth (Hrsg.): Jacob und Wilhelm Grimm über das Deutsche. Schriften zur Zeit-, Rechts-, Sprach- und Literaturgeschichte. Leipzig. S. 209-220.

Lenz, Alexandra N. (Hrsg.) (2016): German abroad. Perspektiven der Variationslinguistik, Sprachkontakt- und Mehrsprachigkeitsforschung. Göttingen. (= Wiener Arbeiten zur Linguistik 4). 